\title{
Thermodynamic versus kinetic approach to polarization-vorticity coupling
}

\author{
Wojciech Florkowski, ${ }^{1,2, *}$ Avdhesh Kumar, ${ }^{1, \dagger}$ and Radoslaw Ryblewski ${ }^{1, \ddagger}$ \\ ${ }^{1}$ Institute of Nuclear Physics, Polish Academy of Sciences, PL-31342 Krakow, Poland \\ ${ }^{2}$ Jan Kochanowski University, PL-25406 Kielce, Poland
}

(Received 25 June 2018; published 24 October 2018)

\begin{abstract}
We critically compare thermodynamic and kinetic approaches that have been recently used to study relations between the spin polarization and fluid vorticity in systems consisting of spin- $1 / 2$ particles. The thermodynamic approach refers to general properties of global thermal equilibrium with a rigidlike rotation and demonstrates that the spin-polarization and thermal-vorticity tensors are equal. On the other hand, the kinetic approach uses the concept of the Wigner function and its semiclassical expansion. In most of the works done so far, the Wigner functions satisfy kinetic equations with a vanishing collision term. We show that this assumption restricts significantly the applicability of such frameworks and, in contrast to many claims found in the literature, does not allow for drawing any conclusions regarding the relation between the thermal-vorticity and spin-polarization tensors, except for the fact that the two should be constant in global equilibrium. We further show how the kinetic-theory equations including spin degrees of freedom can be used to formulate a hydrodynamic framework for particles with spin. We define hydrodynamic equations starting separately from the formulation by de Groot, van Leeuwen, and van Weert and from the canonical formalism. In the former case the energy-momentum tensor is symmetric and the spin tensor is conserved, while in the latter case the energy-momentum tensor is not symmetric and the spin tensor is not conserved. Nevertheless, in the two cases the total angular momentum is always conserved. Interestingly, the two approaches are connected by the pseudo-gauge transformation, which we explicitly define.
\end{abstract}

DOI: 10.1103/PhysRevC.98.044906

\section{INTRODUCTION}

Recently, in connection with the first positive measurements of the $\Lambda$-hyperon spin polarization [1,2], a lot of interest has been triggered in theoretical studies analyzing the spin polarization and vorticity formation in heavy-ion collisions. One expects that the spin polarization can be related to the global rotation of the strongly interacting matter created in the noncentral collisions, in a way similar to the magnetomechanical Barnett effect [3] and Einstein-de Haas effect [4]. Vorticity can also give rise to new phenomena such as the chiral vortical effect [5,6]. Interestingly, the longitudinal polarization of $\bar{\Lambda}$ was discussed already in the 1980s by Jacob and Rafelski in connection with quark-gluon plasma formation [7]. However, the negative results were reported by the first heavy-ion experiments that measured the $\Lambda$ spin polarization in Dubna [8], at CERN [9], and at BNL [10].

In the context of various effects associated with the spin polarization and vorticity, many theoretical studies have been

\footnotetext{
*wojciech.florkowski@ifj.edu.pl

${ }^{\dagger}$ avdhesh.kumar@ifj.edu.pl

${ }^{\ddagger}$ radoslaw.ryblewski@ifj.edu.pl
}

Published by the American Physical Society under the terms of the Creative Commons Attribution 4.0 International license. Further distribution of this work must maintain attribution to the author(s) and the published article's title, journal citation, and DOI. Funded by $S C O A P^{3}$. performed that refer to the spin-orbit coupling [11-14], statistical properties of matter in equilibrium [15-21], and kinetic models with spin [22-25]. Moreover, closely related works on hydrodynamics with triangle anomalies $[5,26]$ and on the Lagrangian formulation of hydrodynamics [27-29] have been reported.

A natural framework for dealing simultaneously with polarization and vorticity would be relativistic hydrodynamics of polarized fluids. An example of such a framework was recently proposed in Refs. [30,31]. It is based on the local equilibrium distribution functions for particles and antiparticles with spin $1 / 2$, in the form introduced in Ref. [19]. This framework can describe the full space-time evolution of the spin polarization in systems created in high-energy nuclear collisions. We note that the inclusion of the spin degrees of freedom into a hydrodynamic approach represents one of several novel developments in relativistic hydrodynamics which forms the basis for our understanding of the spacetime evolution of matter created in heavy-ion collisions (for recent reviews on progress in relativistic hydrodynamics see Refs. [32,33]).

In this paper we perform a detailed comparison of the thermodynamic and kinetic approaches which deal with the phenomenon of polarization-vorticity coupling in heavy-ion collisions. On the one hand, by the thermodynamic approach we mean a series of papers by Becattini and his collaborators [17-20,34-36], where the authors analyze predominantly the properties of matter in global equilibrium with a rigid rotation. On the other hand, by the kinetic approach we mean 
here Refs. [22-25], where collisionless kinetic equations for the Wigner functions of spin-1/2 particles have been studied.

Similarly to Refs. [22-25] we perform herein a semiclassical expansion of the Wigner function. This method was successfully used in the past (see, for example, Refs. [37-41]) to construct a classical limit of quantum kinetic equations, which yields dynamic equations for both the phase-space distribution functions and the spin phase-space densities. The novel feature of our present work is that we use the form of the equilibrium functions for particles with spin $1 / 2$, proposed in Ref. [19], as an input for the semiclassical expansion. In this way, we can check directly how the thermodynamic and kinetic frameworks are complementary to each other and what one approach implies for the other one.

In order to make our formalism as simple as possible, and to concentrate primarily on the relation between the spin polarization and vorticity, we neglect in this work the effects of the electromagnetic and other mean fields. The inclusion of such fields is left for a separate analysis.

One of our findings is that recent formulations of the kinetic theory [22-25] do not imply the spin-polarization induction by the vorticity. Although there exist solutions of the kinetic equations where the two phenomena are interconnected, they are in general independent. This is due to the fact that the collision term is neglected in such frameworks and the collisionless kinetic equation alone cannot imply the growth of polarization due to vorticity. ${ }^{1}$

We further show that the kinetic-theory results demonstrating relations between polarization and vorticity correspond to the exact solutions of the collisionless kinetic equation. Thus, they can be interpreted as a description of global thermodynamic equilibrium. Only in this case the thermodynamic and kinetic results are fully consistent. To clarify this point, besides the concept of global and local equilibrium, we introduce also the ideas of extended global and extended local equilibrium.

Finally, we analyze different possible ways leading from the kinetic theory to the hydrodynamic equations with spin. They are all based on the application of the conservation laws for charge, energy, linear momentum, and angular momentum. Using the semiclassical expansion for the Wigner function, we introduce hydrodynamic equations starting from the kinetic-theory formulation by de Groot, van Leeuwen, and van Weert (GLW) [45], and using directly the canonical formalism [46]. In the GLW case the energy-momentum tensor is symmetric and the spin tensor is conserved, while in the canonical case the energy-momentum tensor is asymmetric and the spin tensor is not conserved (in both cases the total angular momentum is always conserved). Interestingly, the two approaches are connected by the pseudo-gauge transformation, which we have explicitly constructed.

Conventions and notation. Below we use the following conventions and notation for the metric tensor, Levi-Civita's tensor, and the scalar product: $g_{\mu \nu}=\operatorname{diag}(+1,-1,-1,-1)$,

\footnotetext{
${ }^{1}$ We do not discuss here the chiral kinetic theory [42-44] as its relation to the thermodynamic approach of Refs. [17-19] is not known at the moment and requires a separate analysis.
}

$\epsilon^{0123}=-\epsilon_{0123}=1, a \cdot b=g_{\mu \nu} a^{\mu} b^{\nu}=a^{0} b^{0}-\boldsymbol{a} \cdot \boldsymbol{b}$. Throughout the text we use $c=\hbar=k_{B}=1$; however, we explicitly display $\hbar$ in the discussion of the semiclassical expansion of the Wigner function. All calculations are done using the Dirac representation for the $\gamma$ matrices. The operator $\Delta^{\mu \nu}$ projecting on the space orthogonal to the flow vector $u^{\mu}$ is defined as $\Delta^{\mu \nu}=g^{\mu \nu}-u^{\mu} u^{\nu}$.

The Lorentz invariant measure in the momentum space is denoted as $d P$, namely,

$$
d P=\frac{d^{3} p}{(2 \pi)^{3} E_{p}},
$$

where $E_{p}=\sqrt{m^{2}+\boldsymbol{p}^{2}}$ is the on-mass-shell particle energy, and $p^{\mu}=\left(E_{p}, \boldsymbol{p}\right)$. The particle momenta which are not necessarily on the mass shell and appear as arguments of the Wigner functions are denoted by the four-vector $k^{\mu}=\left(k^{0}, \boldsymbol{k}\right)$.

The square brackets denote antisymmetrization, $t^{[\mu \nu]}=$ $\left(t^{\mu \nu}-t^{v \mu}\right) / 2$. The tilde symbol is used to denote dual tensors, which are obtained from the rank-2 antisymmetric tensors by contraction with the Levi-Civita symbol and division by a factor of 2. For example, $\tilde{\omega}_{\mu \nu}$ denotes the dual spin-polarization tensor defined by the equation

$$
\tilde{\omega}_{\mu \nu}=\frac{1}{2} \epsilon_{\mu \nu \alpha \beta} \omega^{\alpha \beta}
$$

where $\omega^{\alpha \beta}$ is the original spin-polarization tensor. The inverse transformation is

$$
\omega^{\rho \sigma}=-\frac{1}{2} \epsilon^{\rho \sigma \mu \nu} \tilde{\omega}_{\mu \nu}
$$

\section{BASIC CONCEPTS AND METHODOLOGY}

\section{A. Spinless particles: Global and local equilibrium}

Before we start our discussion of various effects connected with spin, it is useful to recall basic features of the kinetic theory for spinless particles: In this case, the relativistic Boltzmann equation for the phase-space distribution function $f(x, p)$ contains two terms: the drift term and the collision integral. This can be schematically written as

$$
p^{\mu} \partial_{\mu} f(x, p)=C[f(x, p)] .
$$

The collision integral $C[f]$ vanishes in two special cases: (i) for noninteracting, free-streaming particles and (ii) for global or local thermodynamic equilibrium. In the first case the distribution function satisfies exactly the drift equation $\left(p^{\mu} \partial_{\mu} f_{\mathrm{fs}}(x, p)=0\right)$ describing, unrelated to the present study, the free motion of particles. In the second case, which is of main interest for us, we have to distinguish between the global and local equilibrium.

In the global thermodynamic equilibrium, the equilibrium distribution function $f_{\text {eq }}(x, p)$ satisfies again the equation of the form $p^{\mu} \partial_{\mu} f_{\text {eq }}(x, p)=0$, which leads in this case to the constraints on the hydrodynamic parameters used to specify the form of $f_{\mathrm{eq}}(x, p)$. In particular, the $\beta_{\mu}(x)$ field, defined traditionally as the ratio of the local fluid four-velocity $u_{\mu}(x)$ to the local temperature $T(x)$, satisfies the Killing equation

$$
\partial_{\mu} \beta_{\nu}(x)+\partial_{\nu} \beta_{\mu}(x)=0 .
$$


Equation (5) has the solution of the form ${ }^{2}$

$$
\beta_{\mu}(x)=\beta_{\mu}^{0}+\varpi_{\mu \nu}^{0} x^{\nu},
$$

where the vector $\beta_{\mu}^{0}$ and the antisymmetric tensor $\varpi_{\mu \nu}^{0}$ are constant. For any form of the field $\beta_{\mu}(x)$, we define thermal vorticity as the rotation

$$
\varpi_{\mu \nu}=-\frac{1}{2}\left(\partial_{\mu} \beta_{\nu}-\partial_{\nu} \beta_{\mu}\right) .
$$

Hence, Eqs. (5) and (6) imply that the thermal vorticity in global equilibrium is constant, $\varpi_{\mu \nu}=\varpi_{\mu \nu}^{0}$. Additionally, in global equilibrium the ratio of the chemical potential to the local temperature should be constant, $\xi(x)=\mu(x) / T(x)=$ $\xi^{0}=$ const.

In the case of local equilibrium, the right-hand side of Eq. (4) vanishes, while its left-hand side, strictly speaking, does not. In this case one should add a correction $\delta f$ to the equilibrium function $f_{\text {eq }}$, which describes dissipative phenomena. Nevertheless, if the gradients of local hydrodynamic variables are sufficiently small, the dissipative terms can be neglected. In this case the hydrodynamic variables in $f_{\text {eq }}$ remain unconstrained. To determine them, one adds further assumptions, most commonly that specific moments of Eq. (4) in the momentum space (those that yield the conservation laws for energy, momentum, or charge) vanish. This methodology leads to the perfect-fluid description.

\section{B. Particles with spin}

The treatment of the collisionless kinetic equation for the Wigner function $\mathcal{W}(x, k)$ that includes spin degrees of freedom has many features in common with the simple spinless system discussed above. As the free-streaming case is not interesting, we are left again with essentially two different physics cases which represent global and local thermodynamic equilibrium. Both of them can be analyzed with the help of the equilibrium distribution functions $f^{+}(x, p)$ and $f^{-}(x, p)$, for particles and antiparticles with spin $1 / 2$, introduced by Becattini and collaborators [19]. As the matter of fact, these functions are $2 \times 2$ Hermitian matrices that can be interpreted as spin-density matrices for each value of the space-time position $x$ and momentum $p$. In addition to typical dependence on the hydrodynamic variables $\beta_{\mu}=u_{\mu} / T$ and $\xi=\beta \mu=\mu / T$, they depend also on the antisymmetric spin-polarization tensor $\omega_{\mu \nu}\left(\omega_{\mu \nu}=-\omega_{\nu \mu}\right)$. The equilibrium Wigner function $\mathcal{W}_{\mathrm{eq}}(x, k)$, constructed from the functions $f^{+}(x, p)$ and $f^{-}(x, p)$, also depends on $\beta_{\mu}, \xi$, and $\omega_{\mu \nu}$. Consequently, it turns out that we can distinguish between four rather than two different types of equilibrium. They can be classified as follows:

(1) Global equilibrium. In this case the $\beta_{\mu}$ field is a Killing vector satisfying Eq. (5), $\varpi_{\mu \nu}=-\frac{1}{2}\left(\partial_{\mu} \beta_{\nu}-\partial_{\nu} \beta_{\mu}\right)=$ const, the spin-polarization tensor is constant and agrees with thermal vorticity, $\omega_{\mu \nu}=\varpi_{\mu \nu}$, and in addition $\xi=$ const.

\footnotetext{
${ }^{2}$ The method of solving the Killing equation is presented in Appendix A.
}

(2) Extended global equilibrium. The $\beta_{\mu}$ field is a Killing vector, $\varpi_{\mu \nu}=-\frac{1}{2}\left(\partial_{\mu} \beta_{\nu}-\partial_{\nu} \beta_{\mu}\right)=$ const, and the spin-polarization tensor is constant, but $\omega_{\mu \nu} \neq$ $\varpi_{\mu \nu}, \xi=$ const.

(3) Local equilibrium. The $\beta_{\mu}$ field is not a Killing vector but we still have $\omega_{\mu \nu}(x)=\varpi_{\mu \nu}(x)$; $\xi$ is allowed to depend on space-time coordinates, $\xi=\xi(x)$.

(4) Extended local equilibrium. The $\beta_{\mu}$ field is not a Killing vector and $\omega_{\mu \nu}(x) \neq \varpi_{\mu \nu}(x)$; moreover, $\xi=$ $\xi(x)$.

The global and extended global equilibrium states correspond to the case where $\mathcal{W}_{\text {eq }}(x, k)$ satisfies exactly the collisionless kinetic equations. However, in the local and extended local equilibrium states only certain moments of the kinetic equation for $\mathcal{W}_{\text {eq }}(x, k)$ can be set equal to zero. They can be used to construct perfect-fluid hydrodynamic equations including spin.

We stress that in this work we assume that the collision term vanishes for each type of equilibrium listed above, provided the equilibrium Wigner function $\mathcal{W}_{\text {eq }}(x, k)$ has the form derived from the functions $f^{+}(x, p)$ and $f^{-}(x, p)$. This assumption should be verified in the future by detailed studies of various collision terms for particles with spin. Such studies may also shed new light on the form of the equilibrium distributions. Before the results of such investigations are known, we continue to assume that the collision term vanishes for $\mathcal{W}_{\text {eq }}(x, k)$.

Before we turn to discussion of the kinetic equation for the Wigner function $\mathcal{W}_{\text {eq }}(x, k)$ it is useful to characterize global thermodynamic equilibrium in the framework of relativistic quantum mechanics. This leads to a natural distinction between the global and extended global equilibrium.

\section{GLOBAL THERMODYNAMIC EQUILIBRIUM IN RELATIVISTIC QUANTUM MECHANICS}

In this section we introduce general features of global thermodynamic equilibrium constructed in the framework of relativistic quantum mechanics. We follow here closely the treatment of Zubarev [47] and Becattini [18]. The main object of interest in this approach is a density operator $\hat{\rho}$ defined by the expression

$$
\begin{aligned}
\hat{\rho}(t)= & \exp \left[-\int d^{3} \Sigma_{\mu}(x)\left(\hat{T}^{\mu \nu}(x) b_{\nu}(x)\right.\right. \\
& \left.\left.-\frac{1}{2} \hat{J}^{\mu, \alpha \beta}(x) \omega_{\alpha \beta}(x)-\hat{N}^{\mu}(x) \xi(x)\right)\right] .
\end{aligned}
$$

Here $d^{3} \Sigma_{\mu}$ is an element of a spacelike, three-dimensional hypersurface $\Sigma_{\mu}$. We may assume that it corresponds to a fixed value of the time coordinate. In this case $d^{3} \Sigma_{\mu}=$ $(d V, 0,0,0)$ and $\hat{\rho}$ becomes a function of $t$. The operators $\hat{T}^{\mu \nu}(x), \hat{J}^{\mu, \alpha \beta}(x)$, and $\hat{N}^{\mu}(x)$ are quantum versions of the energy-momentum tensor, angular momentum tensor, and charge current. They obey the following conservation laws:

$$
\begin{aligned}
\partial_{\mu} \hat{T}^{\mu v}(x) & =0, \\
\partial_{\mu} \hat{J}^{\mu, \alpha \beta}(x) & =0, \\
\partial_{\mu} \hat{N}^{\mu}(x) & =0 .
\end{aligned}
$$


Note that $\hat{J}^{\mu, \alpha \beta}(x)$ is asymmetric in the last two indices, $\hat{J}^{\mu, \alpha \beta}(x)=-\hat{J}^{\mu, \beta \alpha}(x)$, and can be, in general, represented as a sum of the orbital and spin parts:

$$
\hat{J}^{\mu, \alpha \beta}(x)=\hat{L}^{\mu, \alpha \beta}(x)+\hat{S}^{\mu, \alpha \beta}(x) .
$$

The orbital part is expressed by the space-time coordinates and the energy-momentum-tensor components

$$
\hat{L}^{\mu, \alpha \beta}(x)=x^{\alpha} \hat{T}^{\mu \beta}(x)-x^{\beta} \hat{T}^{\mu \alpha}(x) .
$$

Using Eqs. (9) and (10) we find

$$
\partial_{\mu} \hat{S}^{\mu, \alpha \beta}(x)=\hat{T}^{\beta \alpha}(x)-\hat{T}^{\alpha \beta}(x) .
$$

Thus, the spin contribution to the angular momentum is usually not conserved-it is conserved only if the energymomentum operator $\hat{T}^{\alpha \beta}(x)$ is symmetric. The functions $b_{v}(x), \omega_{\alpha \beta}(x)$, and $\xi(x)$ are Lagrange multipliers that should be chosen to maximize the system's entropy. Note that $\omega_{\alpha \beta}(x)=-\omega_{\beta \alpha}(x)$ as any symmetric part of $\omega_{\alpha \beta}(x)$ does not give contribution to Eq. (8).

In global thermodynamic equilibrium we require that the operator $\hat{\rho}(t)$ is independent of time. This condition leads to the constraint

$$
\begin{aligned}
\partial_{\mu}\left(\hat{T}^{\mu \nu}(x) b_{v}(x)-\frac{1}{2} \hat{J}^{\mu, \alpha \beta}(x) \omega_{\alpha \beta}(x)-\hat{N}^{\mu}(x) \xi(x)\right) \\
=\hat{T}^{\mu v}(x)\left(\partial_{\mu} b_{v}(x)\right)-\frac{1}{2} \hat{J}^{\mu, \alpha \beta}(x)\left(\partial_{\mu} \omega_{\alpha \beta}(x)\right) \\
\quad-\hat{N}^{\mu}(x) \partial_{\mu} \xi(x)=0 .
\end{aligned}
$$

From this equation we can conclude that the parameters $\xi$ and $\omega_{\alpha \beta}$ are constants, $\xi=\xi^{0}$ and $\omega_{\alpha \beta}=\omega_{\alpha \beta}^{0} \cdot{ }^{3}$ The form of $b_{v}$ depends on the symmetry of the energy-momentum tensor $\hat{T}^{\mu \nu}(x)$. For symmetric $\hat{T}^{\mu \nu}$, we require that $\partial_{\mu} b_{\nu}+\partial_{\nu} b_{\mu}=$ 0 ; hence $b_{v}$ is a Killing vector,

$$
b_{v}=b_{v}^{0}+\delta \omega_{v \rho}^{0} x^{\rho},
$$

where $b_{v}^{0}$ and $\delta \omega_{\nu \rho}^{0}=-\delta \omega_{\rho v}^{0}$ are constants. However, for a not-symmetric (asymmetric) $\hat{T}^{\mu \nu}$ we require that $\partial_{\mu} b_{v}=0$; hence $b_{v}$ must be a constant vector, $b_{v}=b_{v}^{0}$.

Using the decomposition of the angular momentum into the orbital and spin parts [see Eq. (12)], one can show that the two cases discussed above can be expressed by a single form of the density operator:

$$
\begin{aligned}
\hat{\rho}_{\mathrm{EQ}}= & \exp \left[-\int d^{3} \Sigma_{\mu}(x)\left(\hat{T}^{\mu v}(x) \beta_{\nu}(x)\right.\right. \\
& \left.\left.-\frac{1}{2} \hat{S}^{\mu, \alpha \beta}(x) \omega_{\alpha \beta}^{0}-\hat{N}^{\mu}(x) \xi^{0}\right)\right] .
\end{aligned}
$$

For the asymmetric energy-momentum tensor $\beta_{\mu}(x)=b_{\mu}^{0}+$ $\omega_{\mu \gamma}^{0} x^{\gamma}$ (with constant $b_{\mu}^{0}$ and $\omega_{\mu \gamma}^{0}$ ). This implies that $\beta_{\mu}(x)$ is a Killing vector and thermal vorticity defined by Eq. (7) agrees with the spin-polarization tensor $\omega_{\mu \gamma}=\omega_{\mu \gamma}^{0}$. However, for the symmetric energy-momentum tensor $\beta_{\mu}(x)=$

\footnotetext{
${ }^{3}$ We note that if the tensor $\hat{J}^{\mu, \alpha \beta}$ has additional symmetries, for example, it is completely antisymmetric, more general solutions for $\omega_{\alpha \beta}(x)$ may exist.
}

$b_{\mu}^{0}+\left(\delta \omega_{\mu \gamma}^{0}+\omega_{\mu \gamma}^{0}\right) x^{\gamma}$ (with constant $b_{\mu}^{0}, \delta \omega_{\mu \gamma}^{0}$, and $\left.\omega_{\mu \gamma}^{0}\right)$. In this case $\beta_{\mu}(x)$ is again a Killing vector; however, thermal vorticity defined by Eq. (7) does not necessarily agree with the spin-polarization tensor.

Our discussion indicates that, depending on the symmetry of the energy-momentum tensor, we may deal with global or extended global equilibrium, as they have been defined at the end of Sec. II. For completeness, we define the statistical operator for local equilibrium by the same form as Eq. (17),

$$
\begin{aligned}
\hat{\rho}_{\mathrm{eq}}= & \exp \left[-\int d^{3} \Sigma_{\mu}(x)\left(\hat{T}^{\mu \nu}(x) \beta_{v}(x)\right.\right. \\
& \left.\left.-\frac{1}{2} \hat{S}^{\mu, \alpha \beta}(x) \omega_{\alpha \beta}(x)-\hat{N}^{\mu}(x) \xi(x)\right)\right],
\end{aligned}
$$

allowing for an arbitrary form of $\beta_{\mu}(x)$ and $\xi(x)$, and for two options for $\varpi_{\mu \nu}(x)$ : either $\varpi_{\mu \nu}(x)=\omega_{\mu \nu}(x)$ (local equilibrium) or $\varpi_{\mu \nu}(x) \neq \omega_{\mu \nu}(x)$ (extended local equilibrium).

\section{EQUILIBRIUM WIGNER FUNCTIONS}

\section{A. Spin-dependent equilibrium distribution functions}

To include the spin degrees of freedom, the scalar equilibrium distribution functions are generalized to $2 \times 2$ spindensity matrices for each value of the space-time position $x$ and momentum $p$ [45]:

$$
\begin{aligned}
& {\left[f^{+}(x, p)\right]_{r s} \equiv f_{r s}^{+}(x, p)=\frac{1}{2 m} \bar{u}_{r}(p) X^{+} u_{s}(p),} \\
& {\left[f^{-}(x, p)\right]_{r s} \equiv f_{r s}^{-}(x, p)=-\frac{1}{2 m} \bar{v}_{s}(p) X^{-} v_{r}(p) .}
\end{aligned}
$$

Here $m$ is the (anti)particle mass, while $u_{r}(p)$ and $v_{r}(p)$ are Dirac bispinors (with the spin indices $r$ and $s$ running from 1 to 2 ), and the normalizations

$$
\begin{aligned}
& \bar{u}_{r}(p) u_{s}(p)=2 m \delta_{r s}, \quad \sum_{r=1}^{2} u_{\alpha}^{r}(p) \bar{u}_{\beta}^{r}(p)=(\not p+m)_{\alpha \beta}, \\
& \bar{v}_{r}(p) v_{s}(p)=-2 m \delta_{r s}, \quad \sum_{r=1}^{2} v_{\alpha}^{r}(p) \bar{v}_{\beta}^{r}(p)=(\not p-m)_{\alpha \beta} .
\end{aligned}
$$

Note the minus sign and different ordering of spin indices in Eq. (20) compared to Eq. (19). The objects $f^{ \pm}(x, p)$ are $2 \times 2$ Hermitian matrices with the matrix elements defined by Eqs. (19) and (20).

Following Ref. [19], we use the $4 \times 4$ matrices

$$
X^{ \pm}=\exp \left[ \pm \xi(x)-\beta_{\mu}(x) p^{\mu}\right] M^{ \pm},
$$

where

$$
M^{ \pm}=\exp \left[ \pm \frac{1}{2} \omega_{\mu \nu}(x) \Sigma^{\mu \nu}\right] .
$$

In Eqs. (23) and (24) we use the same notation as that introduced in the previous sections, namely, $\beta^{\mu}(x)=u^{\mu}(x) / T(x)$ and $\xi(x)=\mu(x) / T(x)$, with $\mu(x)$ being the chemical potential (connected with a charge that can be identified, for example, with the baryon number or electric charge). The 
quantity $\omega_{\mu \nu}(x)$ is the spin-polarization tensor, while $\Sigma^{\mu \nu}$ is the spin operator expressed in terms of the Dirac $\gamma$ matrices, $\Sigma^{\mu \nu}=(i / 4)\left[\gamma^{\mu}, \gamma^{\nu}\right]$.

For the sake of simplicity, we restrict ourselves to classical Boltzmann statistics in this work. Following Ref. [30] we further assume that the spin polarization tensor $\omega_{\mu \nu}$ satisfies the two conditions ${ }^{4}$

$$
\omega_{\mu \nu} \omega^{\mu \nu} \geqslant 0, \quad \omega_{\mu \nu} \tilde{\omega}^{\mu \nu}=0
$$

In this case we introduce the variables $\zeta$ and $\Omega$ defined by the expression

$$
\zeta=\frac{\Omega}{T}=\frac{1}{2} \sqrt{\frac{1}{2} \omega_{\mu \nu} \omega^{\mu \nu}} .
$$

It turns out (see Ref. [30]) that $\Omega$ plays the role of the chemical potential related with spin. Using Eq. (25) one finds

$$
M^{ \pm}=\cosh (\zeta) \pm \frac{\sinh (\zeta)}{2 \zeta} \omega_{\mu \nu} \Sigma^{\mu \nu}
$$

\section{B. Equilibrium Wigner functions}

The equilibrium phase-space distribution functions $f^{+}(x, p)$ and $f^{-}(x, p)$ can be used to determine explicit expressions for the corresponding equilibrium (particle and antiparticle) Wigner functions. We construct them using the expressions from Ref. [45],

$\mathcal{W}_{\mathrm{eq}}^{+}(x, k)=\frac{1}{2} \sum_{r, s=1}^{2} \int d P \delta^{(4)}(k-p) u^{r}(p) \bar{u}^{s}(p) f_{r s}^{+}(x, p)$

$\mathcal{W}_{\mathrm{eq}}^{-}(x, k)=-\frac{1}{2} \sum_{r, s=1}^{2} \int d P \delta^{(4)}(k+p) v^{s}(p) \bar{v}^{r}(p) f_{r s}^{-}(x, p)$

The total Wigner function is a simple sum of these two contributions:

$$
\mathcal{W}_{\text {eq }}(x, k)=\mathcal{W}_{\text {eq }}^{+}(x, k)+\mathcal{W}_{\text {eq }}^{-}(x, k) .
$$

Using Eqs. (19)-(22) we find

$$
\begin{aligned}
& \mathcal{W}_{\mathrm{eq}}^{+}(x, k)=\frac{1}{4 m} \int d P \delta^{(4)}(k-p)(\not p+m) X^{+}(\not p+m), \\
& \mathcal{W}_{\mathrm{eq}}^{-}(x, k)=\frac{1}{4 m} \int d P \delta^{(4)}(k+p)(\not p-m) X^{-}(\not p-m) .
\end{aligned}
$$

\footnotetext{
${ }^{4}$ Conditions (25) are satisfied in a natural way if only space components $\omega_{i j}$ are different from zero. This happens, for example, in the case of global equilibrium with a rigid rotation. The nonzero $\omega_{0 i}$ components appear, however, for global equilibrium with a constant acceleration along the fluid stream lines (see Refs. [20,48-51]).
}

With the help of Eq. (27) we can further rewrite these equations in the following form:

$$
\begin{aligned}
\mathcal{W}_{\mathrm{eq}}^{+}(x, k)= & \frac{e^{\xi}}{4 m} \int d P e^{-\beta \cdot p} \delta^{(4)}(k-p) \\
& \times\left[2 m(m+\not p) \cosh (\zeta)+\frac{\sinh (\zeta)}{2 \zeta} \omega_{\mu v}(\not p+m)\right. \\
& \left.\times \Sigma^{\mu \nu}(\not p+m)\right], \\
\mathcal{W}_{\mathrm{eq}}^{-}(x, k)= & \frac{e^{-\xi}}{4 m} \int d P e^{-\beta \cdot p} \delta^{(4)}(k+p) \\
& \times\left[2 m(m-\not p) \cosh (\zeta)-\frac{\sinh (\zeta)}{2 \zeta} \omega_{\mu \nu}(\not p-m)\right. \\
& \left.\times \Sigma^{\mu \nu}(\not p-m)\right] .
\end{aligned}
$$

\section{SPINOR DECOMPOSITION OF THE EQUILIBRIUM WIGNER FUNCTION}

\section{A. Clifford-algebra expansion}

The equilibrium Wigner functions $\mathcal{W}_{\text {eq }}^{ \pm}(x, k)$, being $4 \times 4$ matrices satisfying the relations $\mathcal{W}_{\mathrm{eq}}^{ \pm}(x, k)=\gamma_{0} \mathcal{W}_{\mathrm{eq}}^{ \pm}(x, k)^{\dagger} \gamma_{0}$, can be always expanded in terms of the 16 independent generators of the Clifford algebra $[38,46]$,

$$
\begin{aligned}
\mathcal{W}_{\mathrm{eq}}^{ \pm}(x, k)= & \frac{1}{4}\left[\mathcal{F}_{\mathrm{eq}}^{ \pm}(x, k)+i \gamma_{5} \mathcal{P}_{\mathrm{eq}}^{ \pm}(x, k)+\gamma^{\mu} \mathcal{V}_{\mathrm{eq}, \mu}^{ \pm}(x, k)\right. \\
& \left.+\gamma_{5} \gamma^{\mu} \mathcal{A}_{\mathrm{eq}, \mu}^{ \pm}(x, k)+\Sigma^{\mu \nu} \mathcal{S}_{\mathrm{eq}, \mu \nu}^{ \pm}(x, k)\right]
\end{aligned}
$$

The coefficient functions in the equilibrium Wigner function expansion (35) can be obtained by the following traces:

$$
\begin{aligned}
\mathcal{F}_{\mathrm{eq}}^{ \pm}(x, k) & =\operatorname{tr}\left[\mathcal{W}_{\mathrm{eq}}^{ \pm}(x, k)\right], \\
\mathcal{P}_{\mathrm{eq}}^{ \pm}(x, k) & =-i \operatorname{tr}\left[\gamma^{5} \mathcal{W}_{\mathrm{eq}}^{ \pm}(x, k)\right], \\
\mathcal{V}_{\mathrm{eq}, \mu}^{ \pm}(x, k) & =\operatorname{tr}\left[\gamma_{\mu} \mathcal{W}_{\mathrm{eq}}^{ \pm}(x, k)\right], \\
\mathcal{A}_{\mathrm{eq}, \mu}^{ \pm}(x, k) & =\operatorname{tr}\left[\gamma_{\mu} \gamma^{5} \mathcal{W}_{\mathrm{eq}}^{ \pm}(x, k)\right], \\
\mathcal{S}_{\mathrm{eq}, \mu \nu}^{ \pm}(x, k) & =2 \operatorname{tr}\left[\Sigma_{\mu \nu} \mathcal{W}_{\mathrm{eq}}^{ \pm}(x, k)\right] .
\end{aligned}
$$

Using Eqs. (33) and (34) in expressions (36)-(40), and employing the identities for the Dirac matrices (B1)-(B5) (see Appendix B), we find

$$
\begin{aligned}
\mathcal{F}_{\mathrm{eq}}^{ \pm}(x, k) & =2 m \cosh (\zeta) \int d P e^{-\beta \cdot p \pm \xi} \delta^{(4)}(k \mp p) \\
\mathcal{P}_{\mathrm{eq}}^{ \pm}(x, k)= & 0 \\
\mathcal{V}_{\mathrm{eq}, \mu}^{ \pm}(x, k)= & \pm 2 \cosh (\zeta) \int d P e^{-\beta \cdot p \pm \xi} \delta^{(4)}(k \mp p) p_{\mu}, \\
\mathcal{A}_{\mathrm{eq}, \mu}^{ \pm}(x, k)= & -\frac{\sinh (\zeta)}{\zeta} \int d P e^{-\beta \cdot p \pm \xi} \delta^{(4)}(k \mp p) \tilde{\omega}_{\mu \nu} p^{\nu}, \\
\mathcal{S}_{\mathrm{eq}, \mu \nu}^{ \pm}(x, k)= & \pm \frac{\sinh (\zeta)}{m \zeta} \int d P e^{-\beta \cdot p \pm \xi} \delta^{(4)}(k \mp p) \\
& \times\left[\left(p_{\mu} \omega_{\nu \alpha}-p_{\nu} \omega_{\mu \alpha}\right) p^{\alpha}+m^{2} \omega_{\mu \nu}\right] .
\end{aligned}
$$




\section{B. Relations between equilibrium coefficient functions}

Using Eqs. (41)-(45) one can verify that the equilibrium coefficient functions satisfy the following set of constraints:

$$
\begin{aligned}
k^{\mu} \mathcal{V}_{\mathrm{eq}, \mu}^{ \pm}(x, k) & =m \mathcal{F}_{\mathrm{eq}}^{ \pm}(x, k), \\
k_{\mu} \mathcal{F}_{\mathrm{eq}}^{ \pm}(x, k) & =m \mathcal{V}_{\mathrm{eq}, \mu}^{ \pm}(x, k), \\
\mathcal{P}_{\mathrm{eq}}^{ \pm}(x, k) & =0, \\
k^{\mu} \mathcal{A}_{\mathrm{eq}, \mu}^{ \pm}(x, k) & =0, \\
k^{\mu} \mathcal{S}_{\mathrm{eq}, \mu \nu}^{ \pm}(x, k) & =0 . \\
k^{\beta} \tilde{\mathcal{S}}_{\mathrm{eq}, \mu \beta}^{ \pm}(x, k)+m \mathcal{A}_{\mathrm{eq}, \mu}^{ \pm}(x, k) & =0, \\
\epsilon_{\mu \nu \alpha \beta} k^{\alpha} \mathcal{A}_{\mathrm{eq}}^{ \pm \beta}(x, k)+m \mathcal{S}_{\mathrm{eq}, \mu \nu}^{ \pm}(x, k) & =0 .
\end{aligned}
$$

We note that such constraints are fulfilled also by the total Wigner function given by the sum of the particle and antiparticle contributions [see Eq. (30)]. We also note that Eqs. (46)(52) follow from the algebraic structure of the equilibrium Wigner functions and are satisfied for any form of the fields: $\beta_{\mu}(x), \xi(x)$, and $\omega_{\mu \nu}(x)$. Thus, they hold for four different types of equilibrium specified at the end of Sec. II.

\section{SEMICLASSICAL EXPANSION}

In the previous section we introduced the spinor decomposition of the equilibrium Wigner functions and obtained explicit expressions for the equilibrium coefficient functions. Such a decomposition can be naturally used for any Wigner function (describing particles with spin $1 / 2$ ) and, in fact, it was frequently used in the past to derive classical kinetic equations from the underlying quantum field theory [37-41]). In this section we follow closely this approach and write

$$
\begin{gathered}
\mathcal{W}(x, k)=\frac{1}{4}\left[\mathcal{F}(x, k)+i \gamma_{5} \mathcal{P}(x, k)+\gamma^{\mu} \mathcal{V}_{\mu}(x, k)\right. \\
\left.+\gamma_{5} \gamma^{\mu} \mathcal{A}_{\mu}(x, k)+\Sigma^{\mu \nu} \mathcal{S}_{\mu \nu}(x, k)\right] .
\end{gathered}
$$

In the case where the effects of both the mean fields and collisions can be neglected, the Wigner function satisfies the equation of the form

$$
\left(\gamma_{\mu} K^{\mu}-m\right) \mathcal{W}(x, k)=0
$$

Here $K^{\mu}$ is the operator defined by the expression

$$
K^{\mu}=k^{\mu}+\frac{i \hbar}{2} \partial^{\mu} .
$$

Using Eqs. (53) and (55) in Eq. (54) and comparing the real and imaginary parts of the coefficients in the Clifford-algebra basis we obtain two sets of equations. The real parts give

$$
\begin{aligned}
k^{\mu} \mathcal{V}_{\mu}-m \mathcal{F} & =0, \\
\frac{\hbar}{2} \partial^{\mu} \mathcal{A}_{\mu}+m \mathcal{P} & =0, \\
k_{\mu} \mathcal{F}-\frac{\hbar}{2} \partial^{\nu} \mathcal{S}_{\nu \mu}-m \mathcal{V}_{\mu} & =0, \\
-\frac{\hbar}{2} \partial_{\mu} \mathcal{P}+k^{\beta} \tilde{\mathcal{S}}_{\mu \beta}+m \mathcal{A}_{\mu} & =0, \\
\frac{\hbar}{2}\left(\partial_{\mu} \mathcal{V}_{\nu}-\partial_{\nu} \mathcal{V}_{\mu}\right)-\epsilon_{\mu \nu \alpha \beta} k^{\alpha} \mathcal{A}^{\beta}-m \mathcal{S}_{\mu \nu} & =0,
\end{aligned}
$$

while the imaginary parts yield

$$
\begin{aligned}
\hbar \partial^{\mu} \mathcal{V}_{\mu} & =0, \\
k^{\mu} \mathcal{A}_{\mu} & =0, \\
\frac{\hbar}{2} \partial_{\mu} \mathcal{F}+k^{\nu} \mathcal{S}_{\nu \mu} & =0, \\
k_{\mu} \mathcal{P}+\frac{\hbar}{2} \partial^{\beta} \tilde{\mathcal{S}}_{\mu \beta} & =0, \\
\left(k_{\mu} \mathcal{V}_{\nu}-k_{\nu} \mathcal{V}_{\mu}\right)+\frac{\hbar}{2} \epsilon_{\mu \nu \alpha \beta} \partial^{\alpha} \mathcal{A}^{\beta} & =0 .
\end{aligned}
$$

The form of Eqs. (56)-(65) suggests that we can search for solutions for the expansion coefficient functions in the form of the series

$$
\begin{aligned}
\mathcal{F} & =\mathcal{F}^{(0)}+\hbar \mathcal{F}^{(1)}+\hbar^{2} \mathcal{F}^{(2)}+\cdots, \\
\mathcal{P} & =\mathcal{P}^{(0)}+\hbar \mathcal{P}^{(1)}+\hbar^{2} \mathcal{P}^{(2)}+\cdots, \\
\mathcal{V}_{\mu} & =\mathcal{V}_{\mu}^{(0)}+\hbar \mathcal{V}_{\mu}^{(1)}+\hbar^{2} \mathcal{V}_{\mu}^{(2)}+\cdots, \\
\mathcal{A}_{\mu} & =\mathcal{A}_{\mu}^{(0)}+\hbar \mathcal{A}_{\mu}^{(1)}+\hbar^{2} \mathcal{A}_{\mu}^{(2)}+\cdots, \\
\mathcal{S}_{\mu \nu} & =\mathcal{S}_{\mu \nu}^{(0)}+\hbar \mathcal{S}_{\mu \nu}^{(1)}+\hbar^{2} \mathcal{S}_{\mu \nu}^{(2)}+\cdots .
\end{aligned}
$$

\section{A. Zeroth order}

The leading order (the zeroth order in $\hbar$ ) of the real parts gives

$$
\begin{aligned}
k^{\mu} \mathcal{V}_{\mu}^{(0)}-m \mathcal{F}^{(0)} & =0, \\
\mathcal{P}^{(0)} & =0, \\
k_{\mu} \mathcal{F}^{(0)}-m \mathcal{V}_{\mu}^{(0)} & =0, \\
k^{\beta} \tilde{\mathcal{S}}_{\mu \beta}^{(0)}+m \mathcal{A}_{\mu}^{(0)} & =0, \\
\epsilon_{\mu \nu \alpha \beta} k^{\alpha} \mathcal{A}_{(0)}^{\beta}+m \mathcal{S}_{\mu \nu}^{(0)} & =0,
\end{aligned}
$$

while the leading order of the imaginary parts gives ${ }^{5}$

$$
\begin{aligned}
k^{\mu} \mathcal{A}_{\mu}^{(0)} & =0, \\
k^{\nu} \mathcal{S}_{v \mu}^{(0)} & =0, \\
k_{\mu} \mathcal{V}_{\nu}^{(0)}-k_{\nu} \mathcal{V}_{\mu}^{(0)} & =0 .
\end{aligned}
$$

Equations (69)-(76) indicate the coefficients $\mathcal{F}_{(0)}$ and $\mathcal{A}_{(0)}^{\mu}$ may be treated as the basic independent ones, provided $\mathcal{A}_{(0)}^{\mu}$ satisfies the orthogonality condition (74). The coefficient $\mathcal{V}_{(0)}^{\mu}$ is defined by Eq. (71), which gives

$$
\mathcal{V}_{(0)}^{\mu}=\frac{k^{\mu}}{m} \mathcal{F}_{(0)},
$$

and the coefficient $\mathcal{S}_{\mu \nu}^{(0)}$ is obtained from Eq. (73),

$$
\mathcal{S}_{\mu \nu}^{(0)}=-\frac{1}{m} \epsilon_{\mu \nu \alpha \beta} k^{\alpha} \mathcal{A}_{(0)}^{\beta} \text {. }
$$

\footnotetext{
${ }^{5}$ The imaginary part of the scalar zeroth-order part of Eq. (54) vanishes [see Eq. (61)], whereas the imaginary part of the axial-vector zeroth-order part of Eq. (54) gives Eq. (70) [see Eq. (64)]. Therefore, we consider only three equations obtained from the imaginary parts.
} 
Equation (78) leads directly to the dual tensor $\tilde{\mathcal{S}}_{\mu \nu}^{(0)}$ of the form

$$
\tilde{\mathcal{S}}_{\mu \nu}^{(0)}=\frac{1}{m}\left(k_{\mu} \mathcal{A}_{\nu}^{(0)}-k_{\nu} \mathcal{A}_{\mu}^{(0)}\right) .
$$

One can easily check that expressions (77)-(79) solve Eqs. (69)-(73) and Eqs. (74)-(76) if the axial-vector coefficient $\mathcal{A}_{(0)}^{\mu}$ fulfills Eq. (74).

\section{B. First order}

The next-to-leading order (the first order in $\hbar$ ) of the real parts gives

$$
\begin{aligned}
k^{\mu} \mathcal{V}_{\mu}^{(1)}-m \mathcal{F}^{(1)} & =0, \\
\frac{1}{2} \partial^{\mu} \mathcal{A}_{\mu}^{(0)}+m \mathcal{P}^{(1)} & =0, \\
k_{\mu} \mathcal{F}^{(1)}-\frac{1}{2} \partial^{\nu} \mathcal{S}_{\nu \mu}^{(0)}-m \mathcal{V}_{\mu}^{(1)} & =0, \\
-\frac{1}{2} \partial_{\mu} \mathcal{P}_{(0)}+k^{\beta} \tilde{\mathcal{S}}_{\mu \beta}^{(1)}+m \mathcal{A}_{\mu}^{(1)} & =0, \\
\frac{1}{2}\left(\partial_{\mu} \mathcal{V}_{\nu}^{(0)}-\partial_{\nu} \mathcal{V}_{\mu}^{(0)}\right)-\epsilon_{\mu \nu \alpha \beta} k^{\alpha} \mathcal{A}_{(1)}^{\beta}-m \mathcal{S}_{\mu \nu}^{(1)} & =0 .
\end{aligned}
$$

Equation (81) defines the first-order contribution to the pseudoscalar coefficient,

$$
\mathcal{P}^{(1)}=-\frac{1}{2 m} \partial^{\mu} \mathcal{A}_{\mu}^{(0)} .
$$

Similarly, Eq. (82) can be interpreted as the definition of the first-order vector coefficient,

$$
\mathcal{V}_{\mu}^{(1)}=\frac{1}{m}\left(k_{\mu} \mathcal{F}^{(1)}-\frac{1}{2} \partial^{\nu} \mathcal{S}_{\nu \mu}^{(0)}\right),
$$

while Eq. (84) defines the first-order tensor coefficient,

$$
\mathcal{S}_{\mu \nu}^{(1)}=\frac{1}{2 m}\left(\partial_{\mu} \mathcal{V}_{\nu}^{(0)}-\partial_{\nu} \mathcal{V}_{\mu}^{(0)}\right)-\frac{1}{m} \epsilon_{\mu \nu \alpha \beta} k^{\alpha} \mathcal{A}_{(1)}^{\beta} .
$$

By contraction of Eq. (87) with the Levi-Civita tensor we find the dual first-order tensor coefficient,

$\tilde{\mathcal{S}}_{\mu \nu}^{(1)}=\frac{1}{4 m^{2}} \epsilon_{\mu \nu \alpha \beta}\left(k^{\alpha} \partial^{\beta}-k^{\beta} \partial^{\alpha}\right) \mathcal{F}^{(0)}+\frac{1}{m}\left(k_{\mu} \mathcal{A}_{\nu}^{(1)}-k_{\nu} \mathcal{A}_{\mu}^{(1)}\right)$.

Using Eq. (88) in Eq. (83) we find that the first-order axial coefficient should also be orthogonal to $k^{\mu}$, namely, $k_{\mu} \mathcal{A}_{(1)}^{\mu}=0$.

The first-order imaginary parts give

$$
\begin{aligned}
\partial^{\mu} \mathcal{V}_{\mu}^{(0)} & =0, \\
k^{\mu} \mathcal{A}_{\mu}^{(1)} & =0, \\
\frac{1}{2} \partial_{\mu} \mathcal{F}^{(0)}+k^{\nu} \mathcal{S}_{\nu \mu}^{(1)} & =0, \\
k_{\mu} \mathcal{P}^{(1)}+\frac{1}{2} \partial^{\beta} \tilde{\mathcal{S}}_{\mu \beta}^{(0)} & =0, \\
k_{\mu} \mathcal{V}_{\nu}^{(1)}-k_{\nu} \mathcal{V}_{\mu}^{(1)}+\frac{1}{2} \epsilon_{\mu \nu \alpha \beta} \partial^{\alpha} \mathcal{A}_{(0)}^{\beta} & =0 .
\end{aligned}
$$

Combining Eq. (89) with Eq. (77) we find the important formula

$$
k^{\mu} \partial_{\mu} \mathcal{F}_{(0)}(x, k)=0
$$

This is nothing else but the kinetic equation to be satisfied by the scalar coefficient of the Wigner function. Equation (90) confirms that the axial-vector coefficient is orthogonal to $k$ in both the zeroth and first orders. Doing straightforward algebraic manipulations we can check that Eq. (91) is satisfied provided Eq. (94) holds.
Equation (92) leads directly to the kinetic equation obeyed by the axial-vector coefficient,

$$
k^{\mu} \partial_{\mu} \mathcal{A}_{(0)}^{v}(x, k)=0, \quad k_{\nu} \mathcal{A}_{(0)}^{v}(x, k)=0 .
$$

Using Eq. (95) and the orthogonality condition (90) we can check now that Eq. (93) is also satisfied.

\section{Second order}

By studying the zeroth and first orders of the semiclassical expansion we have found that the basic coefficient functions are the scalar and axial-vector components. Their leadingorder terms $\mathcal{F}_{(0)}(x, k)$ and $\mathcal{A}_{(0)}^{v}(x, k)$ satisfy the kinetic equations (94) and (95). The axial-vector coefficient should be (in the zeroth and first orders) orthogonal to the four-vector $k$. If the functions $\mathcal{F}_{(0)}(x, k)$ and $\mathcal{A}_{(0)}^{v}(x, k)$ are known, all other coefficient functions in the zeroth order can be determined through the algebraic relations (70), (77), and (78).

We emphasize that although the system of equations derived above is consistent up to the first order in $\hbar$ (the property demonstrated in several previous studies), it is not sufficient to determine the first-order coefficient functions. We are missing dynamic equations that could be used to determine the evolution of the coefficient functions $\mathcal{F}_{(1)}(x, k)$ and $\mathcal{A}_{(1)}^{v}(x, k)$. This is expected, since we have just seen that the zeroth order is not sufficient to determine the evolution of the functions $\mathcal{F}_{(0)}(x, k)$ and $\mathcal{A}_{(0)}^{v}(x, k)$-this requires going to the first order. Thus, the functions $\mathcal{F}_{(1)}(x, k)$ and $\mathcal{A}_{(1)}^{v}(x, k)$ should be obtained from the analysis of the second order. Such an analysis is completely analogous to that done in the first order and, in fact, leads to the same form of equations:

$$
\begin{aligned}
& k^{\mu} \partial_{\mu} \mathcal{F}_{(1)}(x, k)=0, \\
& k^{\mu} \partial_{\mu} \mathcal{A}_{(1)}^{v}(x, k)=0, \quad k_{\nu} \mathcal{A}_{(1)}^{v}(x, k)=0 .
\end{aligned}
$$

If $\mathcal{F}_{(1)}$ and $\mathcal{A}_{(1)}^{v}$ are determined, the quantities $\mathcal{P}^{(1)}, \mathcal{V}_{\mu}^{(1)}$, and $\mathcal{S}_{\mu \nu}^{(1)}$ are obtained from Eqs. (85), (86), and (87), respectively.

\section{EXACT SOLUTIONS}

It is very interesting to observe that the algebraic structure of the equilibrium coefficient functions, defined by Eqs. (46)(52), is consistent with the zeroth-order equations obtained from the semiclassical expansion of the Wigner function discussed in Sec. VIA [see Eqs. (69)-(76)]. This suggests that the global and extended global equilibrium distributions can be indeed constructed from the functions (33) and (34), provided they fulfill in addition the kinetic equations (94) and (95).

We have to emphasize here, however, that the equilibrium coefficient functions defined by Eqs. (41)-(45) specify only the leading-order terms in $\hbar$ of the "true" equilibrium function that solves the kinetic equation. ${ }^{6}$ To summarize our findings

\footnotetext{
${ }^{6}$ Our approach is based on the form postulated in Ref. [19] that may be missing some important quantum contributions. In particular, the functions $\mathcal{W}_{\text {eq }}(x, k)$ are always on the mass shell; hence, they neglect off-shell quantum propagation of particles.
} 
we can write

$$
\begin{aligned}
& \mathcal{F}^{(0)}=\mathcal{F}_{\text {eq }}, \\
& \mathcal{P}^{(0)}=0, \\
& \mathcal{V}_{\mu}^{(0)}=\mathcal{V}_{\text {eq }, \mu}, \\
& \mathcal{A}_{\mu}^{(0)}=\mathcal{A}_{\text {eq }, \mu}, \\
& \mathcal{S}_{\mu \nu}^{(0)}=\mathcal{S}_{\text {eq }, \mu \nu},
\end{aligned}
$$

in the zeroth order, and similarly

$$
\begin{aligned}
& \mathcal{P}^{(1)}=-\frac{1}{2 m} \partial^{\mu} \mathcal{A}_{\mathrm{eq}, \mu}, \\
& \mathcal{V}_{\mu}^{(1)}=\frac{1}{m}\left(k_{\mu} \mathcal{F}^{(1)}-\frac{1}{2} \partial^{\nu} \mathcal{S}_{\mathrm{eq}, \nu \mu}\right), \\
& \mathcal{S}_{\mu \nu}^{(1)}=\frac{1}{2 m}\left(\partial_{\mu} \mathcal{V}_{\mathrm{eq}, \nu}-\partial_{\nu} \mathcal{V}_{\mathrm{eq}, \mu}\right)-\frac{1}{m} \epsilon_{\mu \nu \alpha \beta} k^{\alpha} \mathcal{A}_{(1)}^{\beta},
\end{aligned}
$$

in the first order.

Let us check now the constraints imposed on the equilibrium coefficient functions by Eqs. (94) and (95). One can easily find that they lead to the equations

$$
\begin{aligned}
k^{\mu} \partial_{\mu} \mathcal{F}_{\text {eq }}(x, k) & =0, \\
k^{\mu} \partial_{\mu} \mathcal{A}_{\text {eq }}^{v}(x, k) & =0, \quad k_{\nu} \mathcal{A}_{\text {eq }}^{v}(x, k)=0 .
\end{aligned}
$$

Using Eqs. (41) and (44) in Eqs. (106) and (107) we conclude that the kinetic equations are exactly fulfilled if the $\beta_{\mu}$ field is the Killing vector defined by Eqs. (5) and (6), while the parameter $\xi$ and the spin-polarization tensor $\omega_{\mu \nu}$ are constant [this implies that the parameter $\zeta$ defined by Eq. (26) is also constant]).

Consequently, the kinetic equations considered in this work (and also in the previous works that used the same mathematical setup) do not constrain the spin-polarization tensor $\omega_{\mu \nu}$ to be equal to the thermal vorticity $\varpi_{\mu \nu}$. In the semiclassical approach discussed here, both tensors should be constant but may be not related to each other. This situation corresponds to extended global equilibrium rather than to global equilibrium. Most likely, the equality of the tensors $\omega_{\mu \nu}$ and $\varpi_{\mu \nu}$ (the fact expected on very general thermodynamic grounds; see Sec. III) could follow from the proper entropy maximization. The present approach, however, does not offer any reliable method for such a calculation. We note that the first-order equations (96) and (97) are decoupled in our equilibrium scheme; thus, we assume below that $\mathcal{F}^{(1)}(x, k)=$ $\mathcal{A}_{\mu}^{(1)}(x, k)=0$.

It is also possible that the relation $\omega_{\mu \nu}=\varpi_{\mu \nu}$ can be necessary for the collision term to vanish. The form of the latter is, however, not known. As we have mentioned above, in this work we assume that any Wigner function of the form (35), with the coefficient functions given by Eqs. (41)-(45), yields a vanishing collision integral.

\section{LOCAL CONSERVATION LAWS}

Having explored consequences of the assumption that the equilibrium Wigner function satisfies exactly the kinetic equation (54), we turn now to a discussion of approximate solutions. Usually, they are obtained by demanding that only certain moments of the kinetic equation (54) yield zero. The selection of such moments for particles with spin is, however, not obvious and one of the aims of this work is to give some insight into this problem. To set the stage, we discuss in this section local conservation laws, which suggest which moments of Eq. (54) may be relevant for construction of the hydrodynamic framework.

\section{A. Charge current}

Expressing the charge current $\mathcal{N}^{\alpha}(x)$ in terms of the Wigner function $\mathcal{W}(x, k)$ we obtain [45]

$$
\mathcal{N}^{\alpha}(x)=\operatorname{tr} \int d^{4} k \gamma^{\alpha} \mathcal{W}(x, k)=\int d^{4} k \mathcal{V}^{\alpha}(x, k) .
$$

In the equilibrium case we use Eqs. (100) and (104) for $\mathcal{V}^{\alpha}(x, k)$. In this way we find

$$
\mathcal{N}_{\mathrm{eq}}^{\alpha}(x)=N_{\mathrm{eq}}^{\alpha}(x)+\delta N_{\mathrm{eq}}^{\alpha}(x),
$$

where

$$
N_{\mathrm{eq}}^{\alpha}(x)=\frac{1}{m} \int d^{4} k k^{\alpha} \mathcal{F}_{\mathrm{eq}}(x, k)
$$

and

$$
\delta N_{\mathrm{eq}}^{\alpha}(x)=-\frac{\hbar}{2 m} \int d^{4} k \partial_{\lambda} \mathcal{S}_{\mathrm{eq}}^{\lambda \alpha}(x, k) .
$$

We have assumed here that $\mathcal{F}^{(1)}(x, k)=0$, which is a trivial solution of the kinetic equation (96).

The charge current should be conserved, which is expressed by the equation

$$
\partial_{\alpha} N_{\mathrm{eq}}^{\alpha}(x)=0 .
$$

Here we used the property $\partial_{\alpha} \delta N_{\mathrm{eq}}^{\alpha}(x)=0$, which follows from the antisymmetry of the tensor $\mathcal{S}_{\mathrm{eq}}^{\lambda \alpha}(x, k)$. One can check that Eq. (112) holds in (extended) global equilibrium, due to Eq. (106). In the (extended) local equilibrium Eq. (112) becomes a condition for the hydrodynamic fields: $\beta_{\mu}(x), \xi(x)$, and $\omega_{\mu \nu}(x)$ that may vary in space and time. Substituting Eq. (41) into Eq. (110) we obtain

$$
N_{\mathrm{eq}}^{\alpha}=4 \cosh (\zeta) \sinh (\xi) \int \frac{d^{3} p}{(2 \pi)^{3} E_{p}} p^{\alpha} e^{-\beta \cdot p}
$$

which agrees with Eq. (12) from Ref. [30]. Doing the integral over the momentum, one finds that the charge current is proportional to the flow vector,

$$
N_{\mathrm{eq}}^{\alpha}=n u^{\alpha},
$$

where

$$
n=4 \cosh (\zeta) \sinh (\xi) n_{(0)}(T)
$$

is the charge density. ${ }^{7}$ Here $n_{(0)}(T)=\langle(u \cdot p)\rangle_{0}$ is the number density of spin- 0 , neutral Boltzmann particles, obtained using

\footnotetext{
${ }^{7}$ One should include also the contribution from Eq. (111) to the charge current. We intend to analyze this issue in a separate paper [52].
} 
the thermal average

$$
\langle\cdots\rangle_{0} \equiv \int \frac{d^{3} p}{(2 \pi)^{3} E_{p}}(\cdots) e^{-\beta \cdot p} .
$$

\section{B. Energy-momentum and spin tensors}

\section{GLW formulation}

Adopting the kinetic-theory framework derived by de Groot, van Leeuwen, and van Weert in Ref. [45], where the energymomentum tensor is expressed directly by the trace of the Wigner function, we can use the following expression:

$$
\begin{aligned}
T_{\mathrm{GLW}}^{\mu \nu}(x) & =\frac{1}{m} \operatorname{tr} \int d^{4} k k^{\mu} k^{\nu} \mathcal{W}(x, k) \\
& =\frac{1}{m} \int d^{4} k k^{\mu} k^{\nu} \mathcal{F}(x, k) .
\end{aligned}
$$

In the equilibrium case, we consider Eq. (117) up to the first order in $\hbar$ using Eq. (98) and setting $\mathcal{F}^{(1)}(x, k)=0$, similarly as in the case of the charge current. Hence, with the help of Eq. (41) we obtain

$$
T_{\mathrm{GLW}}^{\mu \nu}(x)=4 \cosh (\zeta) \cosh (\xi) \int \frac{d^{3} p}{(2 \pi)^{3} E_{p}} p^{\mu} p^{v} e^{-\beta \cdot p} .
$$

In this way we reproduce the perfect-fluid formula given earlier in Ref. [30],

$$
T_{\mathrm{GLW}}^{\mu \nu}(x)=(\varepsilon+P) u^{\mu} u^{\nu}-P g^{\mu \nu},
$$

where the energy density and pressure are given by the expressions

$$
\varepsilon=4 \cosh (\zeta) \cosh (\xi) \varepsilon_{(0)}(T)
$$

and

$$
P=4 \cosh (\zeta) \cosh (\xi) P_{(0)}(T),
$$

respectively. In analogy to the density $n_{(0)}(T)$, we define the auxiliary quantities $\varepsilon_{(0)}(T)=\left\langle(u \cdot p)^{2}\right\rangle_{0}$ and $P_{(0)}(T)=$ $-(1 / 3)\left\langle\left[p \cdot p-(u \cdot p)^{2}\right]\right\rangle_{0}$. The energy-momentum tensor should be conserved; hence, we demand

$$
\partial_{\alpha} T_{\mathrm{GLW}}^{\alpha \beta}(x)=0 .
$$

Similarly to the case of the charge conservation, one can check that Eq. (122) holds in (extended) global equilibrium, provided Eq. (106) is satisfied. Again, in the (extended) local equilibrium Eq. (122) becomes a condition (strictly speaking, four equations) for the hydrodynamic fields: $\beta_{\mu}(x), \xi(x)$, and $\omega_{\mu \nu}(x)$.

The GLW spin tensor has the following form [45]:

$$
\begin{aligned}
S_{\mathrm{GLW}}^{\lambda, \mu \nu}= & \frac{\hbar}{4} \int d^{4} k \operatorname{tr}\left[\left(\left\{\sigma^{\mu \nu}, \gamma^{\lambda}\right\}+\frac{2 i}{m}\left(\gamma^{[\mu} k^{\nu]} \gamma^{\lambda}\right.\right.\right. \\
& \left.\left.\left.-\gamma^{\lambda} \gamma^{[\mu} k^{\nu]}\right)\right) \mathcal{W}(x, k)\right] .
\end{aligned}
$$

For dimensional reasons, we have implemented here the Planck constant. Its presence implies that in equilibrium we may take the leading-order expression for the Wigner function and assume $\mathcal{W}(x, k)=\mathcal{W}_{\text {eq }}(x, k)$. Using Eqs. (33) and (34) in Eq. (123), performing the appropriate traces, and then carrying out the integration over $k$ we get

$$
\begin{aligned}
S_{\mathrm{GLW}}^{\lambda, \mu \nu}= & \frac{\hbar \sinh (\zeta) \cosh (\xi)}{m^{2} \zeta} \int d P e^{-\beta \cdot p} p^{\lambda} \\
& \times\left(m^{2} \omega^{\mu \nu}+2 p^{\alpha} p^{[\mu} \omega^{\nu]}{ }_{\alpha}\right) \\
= & \frac{\hbar w}{4 \zeta} u^{\lambda} \omega^{\mu \nu}+\frac{2 \hbar \sinh (\zeta) \cosh (\xi)}{m^{2} \zeta} s_{\mathrm{GLW}}^{\lambda, \mu \nu},
\end{aligned}
$$

where we have introduced the spin density $w$ defined by the expression [30]

$$
w=4 \sinh (\zeta) \cosh (\xi) n_{(0)}(T),
$$

the auxiliary tensor

$$
\begin{aligned}
s_{\mathrm{GLW}}^{\lambda, \mu \nu}= & A u^{\lambda} u^{\alpha} u^{[\mu} \omega^{\nu]}{ }_{\alpha}+B\left(\Delta^{\lambda \alpha} u^{[\mu} \omega^{\nu]}{ }_{\alpha}+u^{\lambda} \Delta^{\alpha[\mu} \omega^{\nu]}{ }_{\alpha}\right. \\
& \left.+u^{\alpha} \Delta^{\lambda[\mu} \omega^{\nu]}{ }_{\alpha}\right),
\end{aligned}
$$

and the thermodynamic coefficients

$$
\begin{aligned}
B & =-\frac{1}{\beta}\left(\varepsilon_{(0)}+P_{(0)}\right), \quad A=\frac{1}{\beta}\left[3 \varepsilon_{(0)}+\left(3+\frac{m^{2}}{T^{2}}\right) P_{(0)}\right] \\
& =-3 B+\frac{m^{2}}{T} P_{(0)} .
\end{aligned}
$$

Since the energy-momentum tensor derived in Ref. [45] is symmetric, the spin tensor (124) should be also conserved [see, for example, Eq. (14)]:

$$
\partial_{\lambda} S_{\mathrm{GLW}}^{\lambda, \mu v}(x)=0 .
$$

This formula implies that the angular momentum conservation holds separately for the orbital and spin parts.

At this point, it is important to stress that the coefficient function $\mathcal{F}_{\text {eq }}(x, k)$ involves all hydrodynamic variables, i.e., $\beta_{\mu}, \xi$, and the spin-polarization tensor $\omega_{\mu \nu}$-altogether 11 independent functions. This makes the system of Eqs. (112) and (122) insufficient to determine their space-time dependence unless some other information is taken into account. One possibility is to assume a local equilibrium state as defined at the end of Sec. II (the third point). In this case the spin-polarization tensor is equal to the thermal vorticity and the number of independent equations becomes equal to the number of unknown functions. However, since the spin-polarization tensor depends on the space-time gradients of the field $\beta_{\mu}$ in this case, the conservation laws become second-order partial differential equations. Clearly, they do not resemble standard hydrodynamic equations and it is not obvious at the moment how one can treat and solve them. Another possibility is to introduce extended local equilibrium (the fourth point discussed at the end of Sec. II) and to treat the spin-polarization tensor and thermal vorticity as independent quantities. The evolution of the $\omega_{\mu \nu}$ components should follow from the angular momentum conservation, which for the case discussed in this section is reduced to Eq. (128). This approach was proposed originally in Ref. [30] with a phenomenological version of the spin tensor that agrees with the first term in the second line of Eq. (124). 


\section{Canonical version}

The canonical forms of the energy-momentum and spin tensors, $T_{\text {can }}^{\mu \nu}(x)$ and $S_{\text {can }}^{\lambda, \mu \nu}(x)$, can be obtained directly from the Dirac Lagrangian by applying the Noether theorem [46]:

$$
T_{\text {can }}^{\mu \nu}(x)=\int d^{4} k k^{\nu} \mathcal{V}^{\mu}(x, k)
$$

and

$$
\begin{aligned}
S_{\mathrm{can}}^{\lambda, \mu \nu}(x) & =\frac{\hbar}{4} \int d^{4} k \operatorname{tr}\left[\left\{\sigma^{\mu \nu}, \gamma^{\lambda}\right\} \mathcal{W}(x, k)\right] \\
& =\frac{\hbar}{2} \epsilon^{\kappa \lambda \mu \nu} \int d^{4} k \mathcal{A}_{\kappa}(x, k) \equiv \frac{\hbar}{2} \epsilon^{\kappa \lambda \mu \nu} \mathcal{A}_{\kappa}(x) .
\end{aligned}
$$

Here we have used the anticommutation relation $\left\{\sigma^{\mu \nu}, \gamma^{\lambda}\right\}=$ $-2 \epsilon^{\mu \nu \lambda \kappa} \gamma_{\kappa} \gamma_{5}$ to express directly the canonical spin tensor by the axial-vector coefficient function $\mathcal{A}_{\kappa}(x, k)$.

Including the components of $\mathcal{V}^{\mu}(x, k)$ up to the first order in the equilibrium case we obtain

$$
T_{\text {can }}^{\mu \nu}(x)=T_{\mathrm{GLW}}^{\mu \nu}(x)+\delta T_{\mathrm{can}}^{\mu \nu}(x),
$$

where

$$
\delta T_{\text {can }}^{\mu \nu}(x)=-\frac{\hbar}{2 m} \int d^{4} k k^{\nu} \partial_{\lambda} \mathcal{S}_{\mathrm{eq}}^{\lambda \mu}(x, k)=-\partial_{\lambda} S_{\mathrm{GLW}}^{\nu, \lambda \mu}(x) .
$$

The canonical energy-momentum tensor should be exactly conserved; hence, in analogy to Eq. (122), we require

$$
\partial_{\alpha} T_{\text {can }}^{\alpha \beta}(x)=0 .
$$

It is interesting to observe that the conservation laws (122) and (133) are consistent, since $\partial_{\mu} \delta T_{\text {can }}^{\mu \nu}(x)=0$. The latter property follows directly from the definition of $\delta T_{\text {can }}^{\mu \nu}(x)$ [see Eq. (132)].

For the equilibrium spin tensor it is enough to consider the axial-vector component in Eq. (130) in the zeroth order, $\mathcal{A}_{\kappa}^{(0)}(x, k)=\mathcal{A}_{\mathrm{eq}, \kappa}(x, k)$. Then, using Eq. (44) in Eq. (130) and carrying out the integration over the four-momentum $k$ we get

$$
\begin{aligned}
S_{\mathrm{can}}^{\lambda, \mu \nu}= & \frac{\hbar \sinh (\zeta) \cosh (\xi)}{\zeta} \int d P e^{-\beta \cdot p} \\
& \times\left(\omega^{\mu \nu} p^{\lambda}+\omega^{\nu \lambda} p^{\mu}+\omega^{\lambda \mu} p^{\nu}\right) \\
= & \frac{\hbar w}{4 \zeta}\left(u^{\lambda} \omega^{\mu \nu}+u^{\mu} \omega^{\nu \lambda}+u^{\nu} \omega^{\lambda \mu}\right) \\
= & S_{\mathrm{GLW}}^{\lambda, \mu \nu}+S_{\mathrm{GLW}}^{\mu, \nu \lambda}+S_{\mathrm{GLW}}^{\nu, \lambda \mu} .
\end{aligned}
$$

It is interesting to notice that the energy-momentum tensor (131) is not symmetric. In such a case, the spin tensor is not conserved and its divergence is equal to the difference of the energy-momentum components. For the case discussed in this section we obtain

$$
\partial_{\lambda} S_{\mathrm{can}}^{\lambda, \mu \nu}(x)=T_{\mathrm{can}}^{\nu \mu}-T_{\mathrm{can}}^{\mu \nu}=-\partial_{\lambda} S_{\mathrm{GLW}}^{\mu, \lambda v}(x)+\partial_{\lambda} S_{\mathrm{GLW}}^{\nu, \lambda \mu}(x) .
$$

One can immediately check, using the last line of Eq. (134), that Eq. (135) is consistent with the conservation of the spin tensor in the GLW approach.

\section{Pseudo-gauge transformation}

In the previous section, we discussed the energymomentum and spin tensors obtained from the canonical formalism and related them to the expressions introduced by de Groot, van Leeuven, and van Weert. In this section we demonstrate that the two versions of tensors are connected by a pseudo-gauge transformation. Indeed, if we introduce the tensor $\Phi^{\lambda, \mu \nu}$ defined by the relation

$$
\Phi^{\lambda, \mu \nu} \equiv S_{\mathrm{GLW}}^{\mu, \lambda \nu}-S_{\mathrm{GLW}}^{\nu, \lambda \mu}
$$

we can write

$$
S_{\mathrm{can}}^{\lambda, \mu \nu}=S_{\mathrm{GLW}}^{\lambda, \mu \nu}-\Phi^{\lambda, \mu \nu}
$$

and

$$
T_{\mathrm{can}}^{\mu \nu}=T_{\mathrm{GLW}}^{\mu \nu}+\frac{1}{2} \partial_{\lambda}\left(\Phi^{\lambda, \mu \nu}+\Phi^{\mu, \nu \lambda}+\Phi^{\nu, \mu \lambda}\right) .
$$

Here, we have used the property that both $S_{\mathrm{GLW}}^{\lambda, \mu \nu}$ and $\Phi^{\lambda, \mu \nu}$ are antisymmetric with respect to exchange of the last two indices. Equations (137) and (138) are an example of the pseudogauge transformation discussed widely in the literature [53]. The most common use of such a transformation is connected with a change from the canonical formalism to the Belinfante one [54] -it provides a symmetric energy-momentum tensor and eliminates completely the spin tensor. In a very recent work, it was argued that the use of tensors that differ by the pseudo-gauge transformation leads to different predictions for measurable quantities such as spectrum and polarization of particles [55]. The results presented in this work can be useful to study such effects in more detail within explicitly defined hydrodynamic models.

\section{Hydrodynamics from moments of the kinetic equations}

In this section we analyze finally the issue connected with the construction of the hydrodynamic framework from the kinetic theory; namely, we try to answer the question of which moments of the kinetic equations should be included to derive hydrodynamic equations. As far as we concentrate on the charge, energy, and momentum conservations, the answer is known-we should consider the zeroth and first moments of the kinetic equation

$$
k^{\mu} \partial_{\mu} \mathcal{F}_{\text {eq }}(x, k)=0 .
$$

In this way we obtain Eq. (112) and Eq. (122).

In any case, the conservation laws for charge, energy, and momentum are not sufficient to determine the dynamics of spin and they should be supplemented by information coming from the equation for the axial coefficient of the equilibrium Wigner function. The latter can be rewritten in the following form:

$$
\begin{aligned}
0= & k^{\alpha} \partial_{\alpha} \int d P e^{-\beta \cdot p} \frac{\sinh (\zeta)}{\zeta}\left[\delta^{(4)}(k-p) e^{\xi}\right. \\
& \left.+\delta^{(4)}(k+p) e^{-\xi}\right] \tilde{\omega}_{\mu \nu} p^{\nu}
\end{aligned}
$$


If we multiply the first line of Eq. (140) by the four-vector $k_{\beta}$, contract it with the Levi-Civita tensor $\epsilon^{\mu \beta \gamma \delta}$, and then integrate the resulting equation again over $k$, we obtain the conservation of the spin tensor in the GLW version [see Eq. (128)]. ${ }^{8}$ This observation suggests that the form of the spin tensor derived by de Groot, van Leeuwen, and van Weert is, in fact, a very natural choice for the hydrodynamic treatment of spin. This would also indicate that one should make an attempt to derive hydrodynamic equations with spin using the GLW expression for the spin tensor. This can be done in a similar way as in Ref. [30]. However, it is not obvious at the moment how Eq. (124) can be included in a consistent construction of the hydrodynamic picture [52].

We close this section with a remark concerning the hydrodynamic equations used in Ref. [22]. Equations (13) and (14) from this work imply that the flow vector $u^{\mu}$ satisfies the Killing equation; hence, it is constant (see the end of Appendix A). Consequently, the vorticity considered in this work is zero and no conclusions about the vorticitypolarization coupling can be drawn from the analysis presented in Ref. [22].

\section{SUMMARY AND CONCLUSIONS}

In this work we compared thermodynamic and kinetic approaches used to study relations between the spin-polarization tensor and fluid vorticity in systems consisting of spin- $1 / 2$ particles. We first discussed the thermodynamic approach that refers to general properties of global thermal equilibrium with a rigidlike rotation. Such a framework demonstrates directly that the spin-polarization and thermal-vorticity tensors are indeed equal in global equilibrium (for asymmetric energymomentum tensors). Then, we turned to the discussion of the kinetic approach based on the concept of the semiclassical expansion of the Wigner function. We analyzed in more detail the case where the Wigner functions satisfy kinetic equations with a vanishing collision term. We have found, in contrast to many earlier claims found in the literature, that this approach does not imply a direct relation between the thermal vorticity and spin polarization, except for the fact that the two should be constant in global equilibrium (we have dubbed this state an extended global equilibrium).

Finally, we outlined procedures for obtaining hydrodynamic equations from the kinetic equations with spin. In the GLW case the energy-momentum tensor is symmetric and the spin tensor is conserved, while in the canonical case the energy-momentum tensor has an antisymmetric part and the spin tensor is not conserved. Nevertheless, in these two cases the total angular momentum is always conserved. We also found that the two approaches are connected by the pseudogauge transformation, which we explicitly constructed. This

\footnotetext{
${ }^{8}$ We recall that in the derivation of the hydrodynamic equations we do not assume that the kinetic equations are fulfilled but expect that their specific moments vanish. We also note that the choice of the moments is not obvious. Some hints in this respect can be obtained, for example, by comparing exact solutions of the kinetic equations with the hydrodynamic equations (for example, see Ref. [56]).
}

observation opens up new perspectives for studies of hydrodynamics with spin. From a broader point of view we notice that the classical part of the canonical energy-momentum tensor is symmetric; hence, it is suitable for use in the context of the general theory of relativity, which is a classical theory.

Our results fill the gap between two apparently different approaches to study polarization. They indicate the importance of inclusion of the collision term in the kinetic calculations involving the Wigner function. This may shed light on the form of the equilibrium distribution (Wigner) functions in connection with the entropy production processes. The open question remains as to what extent the equilibrium distribution functions used in this work remain a good approximation to more accurate, quantum equilibrium Wigner functions (with particles being not necessarily on the mass shell).

\section{ACKNOWLEDGMENTS}

We thank F. Becattini and E. Speranza for many illuminating discussions. This work was supported in part by the Polish National Science Center Grant No. 2016/23/B/ST2/00717.

\section{APPENDIX A: KILLING EQUATION}

In this section, for completeness of our presentation, we demonstrate that all solutions of Eq. (5) have the form of Eq. (6) with $b_{\mu}$ and $\varpi_{\mu \nu}$ being constant. We start by differentiation of Eq. (5) with respect to coordinate $x^{\alpha}$. In this way we obtain

$$
\beta_{\nu, \mu \alpha}+\beta_{\mu, v \alpha}=0
$$

where, $\mu \alpha$ denotes differentiation first with respect to the coordinate $x^{\mu}$ and then with respect to the coordinate $x^{\alpha}$. Changing $v \rightarrow \alpha, \mu \rightarrow \nu$, and $\alpha \rightarrow \mu$, we rewrite Eq. (A1) as

$$
\beta_{\alpha, v \mu}+\beta_{\nu, \alpha \mu}=0 \text {. }
$$

Changing $\alpha \rightarrow \mu, v \rightarrow \alpha$, and $\mu \rightarrow v$ in Eq. (A2) we find

$$
\beta_{\mu, \alpha \nu}+\beta_{\alpha, \mu \nu}=0 \text {. }
$$

Introducing the notation $a=\beta_{\nu, \mu \alpha}, b=\beta_{\mu, \nu \alpha}$, and $c=\beta_{\alpha, \mu \nu}$, and using the fact that mixed derivatives are equal, Eqs. (A1)(A3) can be rewritten as a simple system of algebraic equations: $a+b=0, c+a=0$, and $b+c=0$, which has the solution $a=b=c=0$. This implies that the field $\beta_{\mu}$ is a linear function of the coordinates $x^{\nu}$ :

$$
\beta_{\mu}=b_{\mu}+\varpi_{\mu \nu} x^{\nu} .
$$

Using the Killing equation (5) we finally find that $\varpi_{\mu \nu}$ is antisymmetric: $\varpi_{\mu \nu}=-\varpi_{\nu \mu}$.

The $\beta_{\mu}$ field is usually defined by the ratio $u_{\mu} / T$, where $T$ is a local temperature. Thus, in the case $T=$ const, Eq. (5) implies that the four-velocity $u_{\mu}$ itself should be a Killing vector. Writing the solution of this equation as $u_{\mu}=u_{\mu}^{0}+\alpha_{\mu \rho}^{0} x^{\rho}$, where $\alpha_{\mu \rho}^{0}=-\alpha_{\rho \mu}^{0}$ is an antisymmetric tensor with constant components, and using the normalization condition for the four-velocity, we find that $u_{\mu}^{0} u_{0}^{\mu}=1$, 
$u_{\mu}^{0} \alpha_{0}^{\mu \rho}=0$, and $\alpha_{\rho \mu}^{0} \alpha_{0}^{\mu \tau}=0$. These equations imply that $\alpha_{\mu \nu}^{0}=0$, which can be easily checked first in the frame where $u_{\mu}^{0}=(1,0,0,0)$. If the tensor $\alpha_{\mu \nu}^{0}$ vanishes in this frame it means that it is zero in all other frames. Consequently, the flow is not vortical in this case.

\section{APPENDIX B: TRACES OF $\gamma$ MATRICES}

In this section we collect useful results on the traces of products of the Dirac matrices which appear in our formalism. We use the Itzykson-Zuber conventions with $\operatorname{tr}\left(\gamma_{5} \gamma^{\alpha} \gamma^{\beta} \gamma^{\gamma} \gamma^{\delta}\right)=-4 i \epsilon^{\alpha \beta \gamma \delta}$, where $\epsilon^{0123}=+1$ [46]. The identities used to obtain the spinor decomposition of the equilibrium Wigner functions are

$$
\begin{aligned}
\operatorname{tr}\left[(\not p \pm m) \Sigma^{\mu v}(\not p \pm m)\right] & =0, \\
\operatorname{tr}\left[\gamma_{5}(\not p \pm m) \Sigma^{\mu v}(\not p \pm m)\right] & =0,
\end{aligned}
$$

$$
\begin{aligned}
\operatorname{tr}\left[\gamma^{\alpha}(\not p \pm m) \Sigma^{\mu \nu}(\not p \pm m)\right] & =0, \\
\operatorname{tr}\left[\gamma_{\alpha} \gamma_{5}(\not p \pm m) \Sigma_{\rho \sigma}(\not p \pm m)\right] & = \pm 4 m p^{\beta} \epsilon_{\beta \alpha \rho \sigma},
\end{aligned}
$$

and

$$
\begin{aligned}
\operatorname{tr} & {\left[2 \Sigma^{\alpha \beta}(\not p \pm m) \Sigma^{\mu \nu}(\not p \pm m)\right] } \\
= & 4 m^{2}\left(g^{\alpha \mu} g^{\beta \nu}-g^{\alpha \nu} g^{\beta \mu}\right)+4\left(g^{\alpha \nu} p^{\beta} p^{\mu}-g^{\alpha \mu} p^{\beta} p^{v}\right. \\
& \left.+p^{\alpha} p^{v} g^{\beta \mu}-p^{\alpha} p^{\mu} g^{\beta \nu}\right) .
\end{aligned}
$$

To derive Eq. (B5) it is useful to use

$$
\begin{aligned}
& \operatorname{tr}\left(\gamma^{\alpha} \gamma^{\beta} \not p \gamma^{\mu} \gamma^{\nu} \not p\right) \\
&= 2 m^{2}\left(g^{\alpha \beta} g^{\mu \nu}-g^{\alpha \mu} g^{\beta \nu}+g^{\alpha \nu} g^{\beta \mu}\right) \\
&+8\left(g^{\alpha \mu} p^{\beta} p^{\nu}-g^{\alpha \nu} p^{\beta} p^{\mu}+p^{\alpha} p^{\mu} g^{\beta \nu}-p^{\alpha} p^{\nu} g^{\beta \mu}\right)
\end{aligned}
$$

and

$$
\operatorname{tr}\left[\Sigma^{\alpha \beta} \Sigma^{\mu \nu}\right]=g^{\alpha \mu} g^{\beta \nu}-g^{\alpha \nu} g^{\beta \mu} .
$$

[1] L. Adamczyk et al. (STAR Collaboration), Global $\Lambda$ hyperon polarization in nuclear collisions: Evidence for the most vortical fluid, Nature (London) 548, 62 (2017).

[2] J. Adam et al. (STAR Collaboration), Global polarization of $\Lambda$ hyperons in $\mathrm{Au}+\mathrm{Au}$ collisions at $\sqrt{s_{N N}}=200 \mathrm{GeV}$, Phys. Rev. C 98, 014910 (2018).

[3] S. J. Barnett, Gyromagnetic and electron-inertia effects, Rev. Mod. Phys. 7, 129 (1935).

[4] A. Einstein and W. de Haas, Experimenteller Nachweis der Ampereschen Molekularstroeme, Verh. Dtsch. Phys. Ges. 17, 152 (1915).

[5] D. E. Kharzeev and D. T. Son, Testing the Chiral Magnetic and Chiral Vortical Effects in Heavy Ion Collisions, Phys. Rev. Lett. 106, 062301 (2011).

[6] D. E. Kharzeev, J. Liao, S. A. Voloshin, and G. Wang, Chiral magnetic and vortical effects in high-energy nuclear collisions—-status report, Prog. Part. Nucl. Phys. 88, 1 (2016).

[7] M. Jacob and J. Rafelski, Longitudinal $\bar{\Lambda}$ polarization, $\bar{\Xi}$ abundance and quark gluon plasma formation, Phys. Lett. B 190, 173 (1987).

[8] M. K. Anikina et al., Characteristics of $\Lambda$ and $\mathrm{K}^{0}$ particles produced in central nucleus nucleus collisions at a $4.5-\mathrm{GeV} / \mathrm{c}$ momentum per incident nucleon, Z. Phys. C 25, 1 (1984).

[9] J. Bartke et al. (NA35 Collaboration), Neutral strange particle production in sulphur sulphur and proton sulphur collisions at 200-GeV/nucleon, Z. Phys. C 48, 191 (1990).

[10] B. I. Abelev et al. (STAR Collaboration), Global polarization measurement in Au+Au collisions, Phys. Rev. C 76, 024915 (2007); 95, 039906(E) (2017).

[11] Z.-T. Liang and X.-N. Wang, Globally Polarized Quark-Gluon Plasma in Noncentral $A+A$ Collisions, Phys. Rev. Lett. 94, 102301 (2005); 96, 039901(E) (2006).

[12] Z.-T. Liang and X.-N. Wang, Spin alignment of vector mesons in non-central A+A collisions, Phys. Lett. B 629, 20 (2005).

[13] J.-H. Gao, S.-W. Chen, W.-T. Deng, Z.-T. Liang, Q. Wang, and X.-N. Wang, Global quark polarization in non-central A+A collisions, Phys. Rev. C 77, 044902 (2008). derivation of differential cross-section in quark-quark scatterings at fixed impact parameter, Front. Phys. China 4, 509 (2009).

[15] C. van Weert, Maximum entropy principle and relativistic hydrodynamics, Ann. Phys. 140, 133 (1982).

[16] D. Zubarev, A. Prozorkevich, and S. Smolyanskii, Derivation of nonlinear generalized equations of quantum relativistic hydrodynamics, Teor. Mat. Fiz. 40, 821 (1979).

[17] F. Becattini and L. Tinti, The ideal relativistic rotating gas as a perfect fluid with spin, Ann. Phys. 325, 1566 (2010).

[18] F. Becattini, Covariant Statistical Mechanics and the StressEnergy Tensor, Phys. Rev. Lett. 108, 244502 (2012).

[19] F. Becattini, V. Chandra, L. Del Zanna, and E. Grossi, Relativistic distribution function for particles with spin at local thermodynamical equilibrium, Ann. Phys. 338, 32 (2013).

[20] F. Becattini and E. Grossi, Quantum corrections to the stressenergy tensor in thermodynamic equilibrium with acceleration, Phys. Rev. D 92, 045037 (2015).

[21] T. Hayata, Y. Hidaka, T. Noumi, and M. Hongo, Relativistic hydrodynamics from quantum field theory on the basis of the generalized Gibbs ensemble method, Phys. Rev. D 92, 065008 (2015)

[22] J.-H. Gao, Z.-T. Liang, S. Pu, Q. Wang, and X.-N. Wang, Chiral Anomaly and Local Polarization Effect from Quantum Kinetic Approach, Phys. Rev. Lett. 109, 232301 (2012).

[23] J.-W. Chen, S. Pu, Q. Wang, and X.-N. Wang, Berry Curvature and Four-Dimensional Monopoles in the Relativistic Chiral Kinetic Equation, Phys. Rev. Lett. 110, 262301 (2013).

[24] R.-H. Fang, L.-G. Pang, Q. Wang, and X.-N. Wang, Polarization of massive fermions in a vortical fluid, Phys. Rev. C 94, 024904 (2016)

[25] R.-H. Fang, J.-Y. Pang, Q. Wang, and X.-N. Wang, Pseudoscalar condensation induced by chiral anomaly and vorticity for massive fermions, Phys. Rev. D 95, 014032 (2017).
[14] S.-W. Chen, J. Deng, J.-H. Gao, and Q. Wang, A General 
[26] D. T. Son and P. Surowka, Hydrodynamics with Triangle Anomalies, Phys. Rev. Lett. 103, 191601 (2009).

[27] D. Montenegro, L. Tinti, and G. Torrieri, The ideal relativistic fluid limit for a medium with polarization, Phys. Rev. D 96, 056012 (2017).

[28] D. Montenegro, L. Tinti, and G. Torrieri, Sound waves and vortices in a polarized relativistic fluid, Phys. Rev. D 96, 076016 (2017).

[29] D. Montenegro and G. Torrieri, Causality and dissipation in relativistic polarizeable fluids, arXiv:1807.02796.

[30] W. Florkowski, B. Friman, A. Jaiswal, and E. Speranza, Relativistic fluid dynamics with spin, Phys. Rev. C 97, 041901 (2018).

[31] W. Florkowski, B. Friman, A. Jaiswal, R. Ryblewski, and E. Speranza, Spin-dependent distribution functions for relativistic hydrodynamics of spin-1/2 particles, Phys. Rev. D 97, 116017 (2018).

[32] W. Florkowski, M. P. Heller, and M. Spalinski, New theories of relativistic hydrodynamics in the LHC era, Rep. Prog. Phys. 81, 046001 (2018).

[33] P. Romatschke and U. Romatschke, Relativistic fluid dynamics in and out of equilibrium-ten years of progress in theory and numerical simulations of nuclear collisions, arXiv:1712.05815.

[34] F. Becattini, L. P. Csernai, D. J. Wang, and Y. L. Xie, $\Lambda$ polarization in peripheral heavy ion collisions, Phys. Rev. C 88, 034905 (2013); 93, 069901(E) (2016).

[35] F. Becattini, I. Karpenko, M. A. Lisa, I. Upsal, and S. A. Voloshin, Global hyperon polarization at local thermodynamic equilibrium with vorticity, magnetic field and feed-down, Phys. Rev. C 95, 054902 (2017).

[36] F. Becattini and I. Karpenko, Collective Longitudinal Polarization in Relativistic Heavy-Ion Collisions at Very High Energy, Phys. Rev. Lett. 120, 012302 (2018).

[37] H. T. Elze, M. Gyulassy, and D. Vasak, Transport equations for the qcd quark wigner operator, Nucl. Phys. B 276, 706 (1986).

[38] D. Vasak, M. Gyulassy, and H. T. Elze, Quantum transport theory for abelian plasmas, Ann. Phys. 173, 462 (1987).

[39] H.-T. Elze and U. W. Heinz, Quark-gluon transport theory, Phys. Rep. 183, 81 (1989).

[40] P. Zhuang and U. W. Heinz, Relativistic quantum transport theory for electrodynamics, Ann. Phys. 245, 311 (1996).
[41] W. Florkowski, J. Hufner, S. P. Klevansky, and L. Neise, Chirally invariant transport equations for quark matter, Ann. Phys. 245, 445 (1996).

[42] M. A. Stephanov and Y. Yin, Chiral Kinetic Theory, Phys. Rev. Lett. 109, 162001 (2012).

[43] J.-Y. Chen, D. T. Son, M. A. Stephanov, H.-U. Yee, and Y. Yin, Lorentz Invariance in Chiral Kinetic Theory, Phys. Rev. Lett. 113, 182302 (2014).

[44] E. V. Gorbar, D. O. Rybalka, and I. A. Shovkovy, Second-order dissipative hydrodynamics for plasma with chiral asymmetry and vorticity, Phys. Rev. D 95, 096010 (2017).

[45] S. de Groot, W. van Leeuwen, and C. van Weert, Relativistic Kinetic Theory: Principles and Applications (North-Holland, Amsterdam, 1980).

[46] C. Itzykson and J. B. Zuber, Quantum Field Theory, International Series in Pure and Applied Physics (McGraw-Hill, New York, 1980).

[47] D. Zubarev, Nonequilibrium Statistical Thermodynamics (Springer, Berlin, 1974).

[48] F. Becattini, Thermodynamic equilibrium with acceleration and the Unruh effect, Phys. Rev. D 97, 085013 (2018).

[49] W. Florkowski, E. Speranza, and F. Becattini, Perfect-fluid hydrodynamics with constant acceleration along the stream lines and spin polarization, Acta Phys. Pol. B 49, 1409 (2018).

[50] G. Prokhorov, O. Teryaev, and V. Zakharov, On the axial current in the rotating and accelerating medium, arXiv:1805.12029.

[51] G. Y. Prokhorov, O. V. Teryaev, and V. I. Zakharov, Effects of rotation and acceleration in the axial current: Density operator vs Wigner function, arXiv:1807.03584.

[52] W. Florkowski, A. Kumar, and R. Ryblewski (unpublished).

[53] F. W. Hehl, On the energy tensor of spinning massive matter in classical field theory and general relativity, Rep. Math. Phys. 9, 55 (1976).

[54] F. J. Belinfante, On the current and the density of the electric charge, the energy, the linear momentum and the angular momentum of arbitrary fields, Physica 7, 449 (1940).

[55] F. Becattini, W. Florkowski, and E. Speranza, Spin tensor and its role in non-equilibrium thermodynamics, arXiv:1807.10994.

[56] L. Tinti, R. Ryblewski, W. Florkowski, and M. Strickland, Testing different formulations of leading-order anisotropic hydrodynamics, Nucl. Phys. A 946, 29 (2016). 